\title{
Manifestaciones psiquiátricas atípicas en el Síndrome
} de Prader-Willi

\section{Atypical psychiatric manifestations in Prader-Willi Syndrome Manifestações psiquiátricas atípicas na síndrome de Prader-Willi}

doi) $\mathrm{http}: / / \mathrm{dx}$.doi.org/10.35954/SM2020.39.1.6

Verónica Santos Spagnuolo a (iD https://orcid.org/0000-0002-9495-4387

(a) Departamento de Salud Mental. Hospital Central de las Fuerzas Armadas.

\section{RESUMEN}

El Síndrome de Prader-Willi es una enfermedad genética rara. La presencia de síntomas psiquiátricos es frecuente, siendo su presentación y evolución atípica. Se realiza un reporte de caso de una paciente de 28 años portadora de Síndrome de Prader-Willi y síntomas psiquiátricos asociados, con evolución hacia la cronicidad.

PALABRAS CLAVE: Demencia; Discapacidad Intelectual; Disomía Uniparental; Síndrome de Prader-Willi; Trastornos Psicóticos.

\section{ABSTRACT}

Prader-Willi Syndrome is a rare genetic disorder. The presences of psychiatric symptoms are frequent, with atypical onset and course. We report a 28 years old woman with Prader-Willi Syndrome and psychiatric symptoms, which developed a chronic course.

KEY WORDS: Dementia; Intellectual Disability; Uniparental Disomy; Prader-Willy Syndrome;

Psychotic Disorders.

\section{RESUMO}

A síndrome de Prader-Willi é uma doença genética rara. A presença de sintomas psiquiátricos é frequente, e a sua apresentação e evolução é atípica. É apresentado um caso de um paciente de 28 anos com Síndrome de Prader-Willi e sintomas psiquiátricos associados, com evolução para a crónica.

Palavras chave: Demência; Deficiência Intelectual; Dissomia Uniparental; Síndrome de Prader-Willi; Trastornos Psicóticos.

\section{INTRODUCCIÓN}

La presencia de síntomas psiquiátricos en personas con enfermedades del neurodesarrollo como el Síndrome de Prader Willi, plantea desafíos clínicos y terapéuticos por su presentación atípica y las dificultades en su tratamiento, sumado a la escasa investigación en la temática dada la baja frecuencia de estos casos.

El Síndrome de Prader-Willi (SPW) es el resultado de la falta de expresión de genes en el cromosoma 15q11-q13, derivado de la madre lo que se conoce como disomía uniparental materna (DUP), o por la deleción de la porción proximal del brazo 
largo del cromosoma 15 paterno (15q11-q13 DEL). Fue descripto en 1956 por Prader, Labhart y Willi, y su prevalencia está estimada en 1 cada 25.000. Su fenotipo conductual se caracteriza por rituales, comportamientos estereotipados y compulsivos. Podrían tener conductas agresivas y auto-lesivas. Discapacidad intelectual moderada y dismorfia (frente estrecha, ojos en forma de almendra, boca triangular) (1).

Dado que la anomalía genética lleva a una alteración del desarrollo del hipotálamo, su disfunción es una característica clave en el SPW. Pudiendo ser considerado un desorden del hipotálamo $(2,3)$. Debido a las disfunciones endócrinas y metabólicas relacionadas con el hipotálamo, el SPW se asocia con un déficit de la Hormona del Crecimiento $(\mathrm{GH})$, reflejado en un leve retraso en el crecimiento prenatal, después del nacimiento baja estatura y falta de crecimiento acelerado en la pubertad. También se ve un descenso de las hormonas sexuales, por una alteración en el funcionamiento de las neuronas liberadoras de hormona luteinizante (LH). Este síndrome también se caracteriza por presentar hiperfagia con aumento de peso, relacionado con la disminución de células que contienen oxitocina ya que esta tiene propiedades anorexígenas. También se ha descrito mal control de la temperatura corporal e hipersomnolencia diurna. Otras características reflejan alteraciones en el funcionamiento del Sistema Nervioso Central (SNC); hipotonía central en la infancia, retraso en el desarrollo psicomotor, dificultades en el desarrollo motor del lenguaje y comportamientos obsesivos compulsivos. Estos pacientes también pueden desarrollar cuadros psicóticos recurrentes, caracterizados por inicio subagudo con recuperación completa e inestabilidad en el estado de ánimo. Se han descrito variadas sintomatologías psiquiátricas que no llegan a configurar diagnósticos claros; estado de ánimo disfórico, cambios cíclicos del estado de ánimo, estados psicóticos de breve duración. Otros autores publicaron sobre 14 pacientes, de los cuales a 10 se le realizaron diagnóstico de psicosis atípica y a 4 trastorno afectivo bipolar (3).
La psicosis se ha caracterizado por síntomas como confusión, alucinaciones acústico-verbales, comportamiento paranoico. Dado que los síntomas psicopatológicos varían longitudinalmente, se recomienda usar el término síndrome psiquiátrico Prader-Willi (2).

\section{CONSIDERACIONES ÉTICAS}

Se solicitó permiso a la paciente y a familiares responsables para escribir el reporte de caso. Se mantiene el anonimato, omitiendo información que pueda ser identificatoria.

\section{CASO CLÍNICO}

Sexo femenino, 28 años, soltera. Vive con sus padres. Actualmente sin actividades académicas o laborales desde hace 7 años. Última actividad cuarto año de secundaria en 2012.

APM: portadora de SPW diagnosticado en agosto del 2010. Patología colónica inflamatoria diagnosticada como Enfermedad de Crohn en 2017, varias internaciones por este motivo.

Antecedentes familiares no presenta.

Antecedentes personales psiquiátricos: primera consulta con psiquiatría por episodio psicótico agudo. Requirió internación en sala de psiquiatría y electroconvulsoterapia (ECT). Ha mantenido controles regulares y tratamiento hasta el presente en la policlínica de psiquiatría del Departamento de Salud Mental del Hospital Central de las Fuerzas Armadas (H.C.FF.AA.). Ha mantenido como tratamiento de base risperidona en dosis terapéuticas de 3 a $4,5 \mathrm{mg} /$ día.

La evolución desde el punto de vista psiquiátrico ha sido atípica y tórpida. Presentando sintomatología polimórfica y atípica dado por:

- Octubre del 2011 a marzo del 2012: episodio psicótico agudo prolongado. Se planteó como desencadenante la utilización de paroxetina. Fue estabilizado con antipsicóticos vía oral, no requiriendo internación. 
- Abril del 2014 a febrero del 2015 inicia conductas compulsivas de la alimentación, con aumento de peso franco $(10 \mathrm{~kg})$. Se estabilizan estas conductas con control por parte de los padres.

- Julio del 2016 reitera conductas compulsivas dado por conductas de robo. Se le agrega fluvoxamina 100 mg/día con mejoría de las mismas.

- Noviembre del 2017 comienza con cuadro psicótico que ha mantenido hasta la actualidad, con mala respuesta a los tratamientos planteados que pasaremos a analizar posteriormente.

La paciente presenta desde el 2017 hasta la fecha un cuadro de inicio insidioso dado por: ideación delirante pobremente elaboradas de daño y perjuicio, autorreferenciales, a mecanismo alucinatorio con soliloquios por momentos, alteración en la forma del pensamiento teniendo un lenguaje que pierde el hilo conductor, sin alteración franca de conciencia. En la evolución agrega un telescopado del tiempo (desorganización cronológica de los eventos sin alteración de memoria), lenguaje con perseveración (repetición de palabras), agrava alteración en la forma del pensamiento comenzando a presentar asociaciones laxas y fabulaciones con lo que empeora la capacidad finalista del lenguaje, tornándose más difícil la comunicación. Franca alteración de las praxias dada por alteración en la planificación de actividades cotidianas, y necesidad de ayuda en su cuidado personal (la tienen que ayudar a vestir). Se plantea en este momento el diagnóstico de episodio psicótico atípico con mala evolución, pudiendo corresponder a un proceso neurodegenerativo subsidiario a su cromosomopatía. En diciembre del 2018 frente a esta mala evolución se realiza interconsulta con colega psiquiatra quien está de acuerdo con el planteo diagnóstico, y sugiere cambio de antipsicótico a aripiprazol. No hubo respuesta favorable al aripiprazol, persistiendo y agravando los síntomas previamente descritos. Evaluada por neurólogo no presentó elementos de neurodegeneración claros.

Se plantea realizar ECT como tratamiento estabilizador frente a esta mala evolución dado el antecedente de buena respuesta en el 2009 con este procedimiento.

- En enero del 2019, se realizó una serie estándar de ECT, con seis sesiones, sin respuesta clínica.

- Marzo del 2019 cambia la presentación clínica agregando elementos de exaltación del humor dado por verborragia llegando a estar disfónica, inquietud psicomotriz e insomnio. Se inició litio a dosis de $600 \mathrm{mg} /$ día y quetiapina llegando a dosis de 400 $\mathrm{mg} /$ día para control de la inquietud psicomotriz. Previa suspensión de aripiprazol. Se estabiliza el cuadro en mayo del 2019, mejorando los elementos de exaltación del humor, persistiendo las alteraciones del pensamiento descritas previamente.

\section{EXAMEN PSIQUIÁTRICO DE JULIO DEL 2019}

Presentación: biotipo típico del SPW: baja estatura, sobrepeso, se presenta en ropas de calle, aliñada, aseada. Edad cronológica mayor a la biológica. Siempre acompañada en consulta por sus padres. Actitud: pueril, participa en la entrevista en la medida de sus posibilidades.

Fascies, mímica y gestualidad: fascies típico del SPW, frente angosta, ojos con forma de almendra, boca triangular. Mímica y gestualidad acorde al relato.

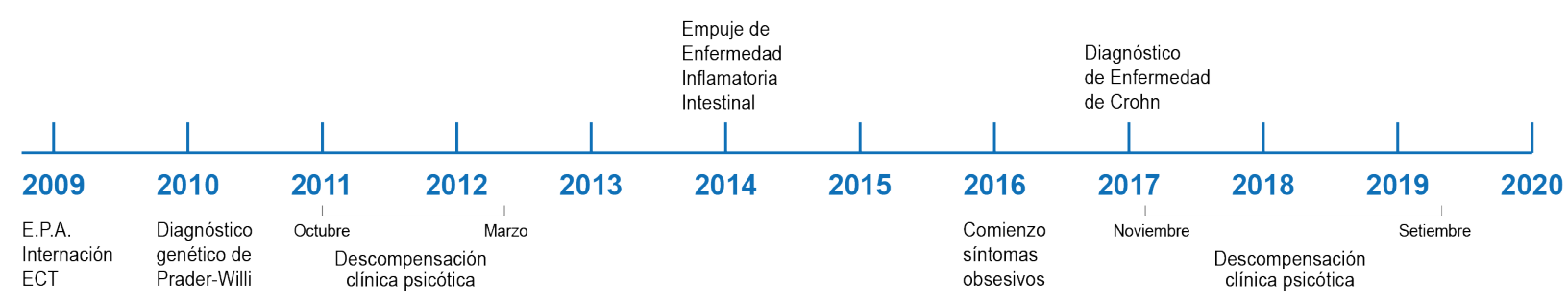

Figura 1. Evolución clínica. 
Conciencia: bien orientada en tiempo y espacio. Atención sin alteraciones. Memoria a largo y corto plazo alterada, dado que no puede organizar cronológicamente los hechos. Presentifica adecuadamente la entrevista.

Rapport: bueno en lo empático, lográndose vínculo afectuoso y cálido. Malo en lo ideico.

Humor y afectividad: eutímica. Afectividad acorde al relato.

\section{Pensamiento}

Forma: curso conservado, sin alteraciones sintácticas, alteración en la prosodia. Discurso que se desorganiza y pierde el hilo conductor, reorganizándose espontáneamente.

Contenido: sin ideas delirantes. Pobre, pueril y concreto, con escaso vuelo abstractivo.

Psicomotricidad: sin alteraciones.

Conductas basales: sueño y apetito conservados con medicación.

Conductas complejas: mantiene alteración en las praxias para la vida diaria.

Nivel intelectual: medido en 2010 por test de nivel informa un coeficiente intelectual de 70 , que corresponde a un nivel intelectual marginal. En la evaluación clínica actual impresiona mucho más descendido.

Personalidad: se puede evaluar como una personalidad que no se ha desarrollado completamente dado el nivel marginal y las dificultades cognitivas propias del SPW. Por lo cual es una personalidad frágil, con dificultades en la adaptabilidad.

\section{PLANTEO CLÍNICO ACTUAL}

La paciente tiene un diagnóstico de planteo desde el punto de vista psiquiátrico, muy difícil de catalogar dentro de las clasificaciones comúnmente utilizadas: la Clasificación Estadística Internacional de Enfermedades y Problemas Relacionados con la Salud, décima revisión (CIE-10) o el Manual Diagnóstico y Estadístico de los Trastornos Mentales (DSM-V), por lo que utilizaremos un diagnóstico clínico-semiológico.
Presenta una alteración del juicio crítico crónico de al menos dos años de evolución por lo cual desde el punto de vista psiquiátrico clínico es una psicosis crónica.

En cuanto a la enfermedad de base que produce esta psicosis crónica es dónde realizamos los planteos diagnósticos:

- Esta psicosis crónica se instala en una paciente con una cromosomopatía que genera alteraciones a nivel del SNC, dado por alteraciones cognitivas y con posibilidad de cuadros psicóticos. Por lo que podría corresponder a la evolución propia del SPW a nivel cerebral, dado por el proceso neurodegenerativo en una paciente con una enfermedad del neurodesarrollo.

- La reiteración de episodios psicóticos agudos, persistiendo luego con una alteración del juicio crítico crónico, con alteración en la forma y contenido del pensamiento en un contexto de alteración de los pragmatismos, comenzando a la edad de 18 años, puede corresponder a un proceso psiquiátrico primario como es la Esquizofrenia.

- La presencia de un descenso en el rendimiento global con alteraciones típicas del deterioro cognitivo como es la alteración en las praxias, la presencia de perseveración en el pensamiento puede orientar a que la falla en el juicio corresponda a un deterioro cognitivo grave pre-senil, lo cual ha sido descrito en estos pacientes (4). La edad tan precoz de inicio, a los 27 años, aleja este planteo, pero no lo descarta.

\section{Estudios paraclínicos realizados}

Diagnóstico genético realizado en 2010, en donde se confirma el diagnóstico de síndrome psiquiátrico Prader-Willi.

Test de nivel intelectual (2010): Cl: 70, nivel marginal. Tomografía Axial de Cráneo con contraste (TAC) (setiembre del 2018), solicitada a causa de la mala evolución del episodio psicótico; que fue informada normal. 
Estudios de rutina: hemograma, glicemia, azoemia, creatininemia, ionograma, funcional y enzimograma hepático, velocidad de eritrosedimentación. Todos dentro de rango normal.

\section{DISCUSIÓN}

EI SPW se asocia con un alto riesgo de problemas psiquiátricos $(1,5)$.

Los pacientes que tienen el defecto genético dado por disomía uniparental materna (DUP) tienen mayor prevalencia de desarrollar una psicosis que aquellos que presentan la delección genética del gen 15q11-q13 (DEL) $(1,6)$.

Hay estudios de prevalencia de psicosis en pacientes portadores de SPW que establece una prevalencia entre el 13 y $62 \%$ de los adultos con DUP y $13 \%$ en adultos con DEL (7-9).

La edad de inicio de los trastornos psicóticos varía entre los 13 y 19 años (1) lo que coincide con la edad de inicio de la sintomatología de la paciente presentada. La edad crítica para el inicio del deterioro conductual y emocional en pacientes con dificultades intelectuales se ha evidenciado como la adolescencia y la adultez temprana (10). Un estudio clínico realizado en los Países Bajos, plantea la hipótesis que los trastornos psiquiátricos, entre ellos la psicosis y depresión, están presentes en niños con SPW y que la prevalencia aumenta con la edad. Así como también que los pacientes portadores de DUP son más proclives a la psicosis en comparación con los que presentan DEL. En este último caso estarían más proclives al desarrollo de síntomas depresivos (5). La publicación realizada por Verhoeven en el año 2006, respecto a la sintomatología más frecuente en los episodios psicóticos en pacientes con SPW, está basado en reporte de casos. Se destaca que son cuadros atípicos y que tienen recidivas. Los síntomas más frecuentemente hallados fueron: ansiedad (95\%), virajes del humor (95\%), confusión (90\%), labilidad emocional (80\%), ideación paranoide $(76 \%)$. Las alucinaciones audi- tivas sólo tuvieron una incidencia del $48 \%$. En este trabajo se enfatiza la remisión total de los síntomas psicóticos, lo cual no ha sucedido en la paciente, Verhoeven también plantea abandonar los diagnósticos categoriales y focalizarse en realizar un tratamiento a largo plazo con finalidad fundamentalmente profiláctica dada la atipicidad de los síntomas. En cuanto al modo de inicio de los trastornos psicóticos en el SPW, se ha descrito que la forma de comienzo más frecuente es la subaguda, sin embargo, en este mismo estudio plantea la atipicidad de los síntomas, así como la remisión total de los mismos luego de estabilizado el cuadro. Si bien la paciente de este reporte sí ha presentado un inicio insidioso (subagudo) de los síntomas, no ha evolucionado a la remisión total de los mismos, permaneciendo con síntomas residuales ya descritos (2).

Con respecto a la presencia de deterioro cognitivo o inicio de una demencia en personas portadoras de SPW, hay poca información. Existe un reporte de caso del año 2010, en el cual se describe el inicio de una demencia en una paciente de 58 años con SPW. El deterioro cognitivo fue grave, perdiendo el lenguaje, la marcha y el control de esfínteres antes de los 56 años. Se hipotetizó la existencia de un envejecimiento prematuro en los portadores de SPW, sin embargo, se necesita mayor investigación al respecto (11).

En referencia al tratamiento la medicación psicotrópica se utiliza ampliamente para el control sintomatológico. En una revisión del año 2015 se plantea la risperidona como antipsicótico que ha demostrado mayor efectividad en el control de los síntomas psicóticos, pero también ha sido vinculado con un mayor aumento de peso. Debido a este aumento de peso producido por la risperidona, hay varios casos publicados sobre el uso de aripiprazol. La ventaja del aripiprazol sobre la risperidona se basa fundamentalmente en que es un antipsicótico que ha sido aprobado para el uso de psicosis en adolescentes y adultos, y que debido al escaso efecto sobre los receptores $\mathrm{H} 1$, 
no produce un aumento significativo de peso (12). Los casos reportados plantean un uso favorable, si bien aclaran que es necesaria mayor investigación al respecto para establecer la eficacia, y presencia de efectos adversos (12). La evolución de nuestra paciente mostró escasa respuesta al tratamiento con aripiprazol, no coincidiendo con lo planteado por Verhoeven (2).

Los estabilizadores del humor que más eficacia han presentado para la estabilización y remisión de los síntomas de exaltación del humor fueron litio y valproato. Así mismo han demostrado eficacia para el control de los síntomas psicóticos y de los trastornos conductuales (2).

\section{CONCLUSIONES}

Las dificultades diagnósticas, así como la atipicidad de los síntomas presentes en la paciente, coinciden con la bibliografía disponible hasta el momento. Los cuadros no son claros, siendo difícil clasificarlos dentro de las nomenclaturas habituales. Con relación a la evolución clínica, la paciente no ha presentado la misma evolución hacia la remisión total del cuadro psicótico como está descrito en los reportes de casos disponibles hasta el momento. En cuanto a la presencia de un deterioro cognitivo prematuro, no hemos encontrado estudios en esa área. La información más aproximada disponible, reporta un caso de una paciente francamente mayor a la que planteamos (58 años).

El tratamiento realizado estuvo de acuerdo con las pautas mencionadas en la bibliografía. Si bien la remisión total de los síntomas psicóticos es lo que está descrito, no ha sido la respuesta que presentó nuestra paciente. En referencia a los síntomas de exaltación del humor, los mismos remitieron en su totalidad con estabilizadores del ánimo, como se plantea en los reportes de casos publicados previamente.

Dada la atipicidad y múltiples síntomas psiquiátricos estamos de acuerdo en plantear el diagnóstico como un síndrome psiquiátrico Prader-Willi (2). La baja frecuencia del SPW hace dificultoso su estudio. La información disponible hasta el momento está basada fundamentalmente en reporte de casos. Los estudios sistematizados y aleatorizados son difíciles por la misma razón. Hace falta más investigación en el área.

Es un desafío para el clínico el diagnóstico y tratamiento de estos pacientes desde un punto de vista psiquiátrico, la formulación de una estrategia a largo plazo, así como su pronóstico.

DECLARACIÓN DE CONFLICTOS DE INTERESES: La autora no reporta ningún conflicto de interés. El estudio se realizó con recursos propios de la autora y/o la institución a la que representa. 


\section{REFERENCIAS}

(1) Clarke D, Boer H, Webb T, Scott P, Frazer S, Vogels A, et al.

Prader-Willi syndrome and psychotic symptoms:

1. Case descriptions and genetic studies.

J Intel Disab Res 1998; 42(Pt 6):440-450.

doi: 10.1046/j.1365-2788.1998.4260440.x

(2) Verhoeven WM, Tuinier S. Prader- Willi syndrome: atypical psychoses and motor dysfunctions. Int Rev Neurobiol 2006; 72:119-30.

doi: 10.1016/S0074-7742(05)72007-9

(3) Bonnot O, Cohen D, Thuilleaux D, Consoli A, Cabal S, Tauber M.

Psychotropic treatments in Prader-Willi syndrome: a critical review of published literature.

Eur J Pediatr 2016; 175(1):9-18.

doi: 10.1007/s00431-015-2670-x

(4) Sinnema M, Schrander-Stumpel CT, Verheij HE, Meeuwsen M, Maaskant MA, Curfs LM.

Dementia in a woman with Prader-Willi syndrome. Eur J Med Genet 2010; 53(3):145-8.

doi: 10.1016/j.ejmg.2010.02.006

(5) Lo S, Collin P, Hokken Koelega A. Psychiatric disorders in children with Prader-Willi syndromeResults of a 2 year longitudinal study.

Am J Med Genet A 2015; 167A(5):983-91.

doi: 10.1002/ajmg.a.36998

(6) Clarke D. Prader-Willi Syndrome and Psychoses. Brit J Psy 1993; 163(5):680-684.

doi: https://doi.org/10.1192/bjp.163.5.680

(7) Vogels A, Hert M, Descheemaeker M, Govers V, Devriendt K, Legius E, et al.

Psychotic disorders in Prader-Willi syndrome.

Am J Med Genet 2004; 127A(3): 238-243.

doi: 10.1002/ajmg.a.30004

(8) Soni S, Whittington J, Holland A, Webb T, Maina E, Boer $\mathrm{H}$, et al.

The phenomenology and diagnosis of psychiatric illness in people with Prader-Willi syndrome.

Psychol Med 2008; 38(10):1505-14.

doi: $10.1017 /$ S0033291707002504
(9) Sinnema M, Boer H, Collin P, Maaskant MA, van Roozendaal KE, Schrander-Stumpel CT, et al. Psychiatric illness in a cohort of adults with

Prader-Willi syndrome.

Res Dev Disabil 2011; 32(5):1729-35.

doi: 10.1016/j.ridd.2011.02.027

(10) Steinhausen HC, Eiholzer U, Hauffa BP, Malin Z. Behavioural and emotional disturbances in people with Prader-Willi syndrome.

J Intellect Disabil Res 2004; 48(1):47-52.

doi: 10.1111/j.1365-2788.2004.00582.x

(11) Ventriglio A, Gentile A, Stella E, Bellomo A. Metabolic issues in patients affected by schizophrenia: clinical characteristics and medical management. Front Neurosci 2015; 9:297.

doi: 10.3389/fnins.2015.00297

(12) Briegel W. Clinical Usefulness of Aripiprazole Treatment in a Girl with Prader-Willi Syndrome and Psychosis.

Clin Psychopharmacol Neurosci 2018; 16(4):497-500. doi: 10.9758/cpn.2018.16.4.497 